\title{
INTEGRAÇÃO SOCIOESPACIAL DOS BAIRROS DE HABITAÇÃO SOCIAL NA ÁREA METROPOLITANA DE LISBOA: EVIDÊNCIAS DE MICRO SEGREGAÇÃO
}

\author{
Marina CARreiras ${ }^{1}$
}

\begin{abstract}
RESUMO - Este artigo reflete sobre a condição urbana dos bairros de habitação social na Área Metropolitana de Lisboa (AML), no que se refere à integração socioespacial e relações desta com o desenvolvimento urbano, as políticas públicas e estratégias locais de atuação nos bairros. Assume-se uma perspetiva multiescalar, que parte de uma análise extensiva dos bairros sociais na AML, para chegar a um estudo intensivo da situação de três bairros localizados em municípios distintos da Grande Lisboa. Para a concretização da investigação, para além da análise bibliográfica, recorreu-se a uma combinação de métodos quantitativos, qualitativos e cartográficos. Os resultados evidenciam lacunas na integração socioespacial dos bairros de promoção municipal a múltiplos níveis geográficos e confirmam a existência de desigualdades socioespaciais enquanto fenómeno multinível que se expressa em diversas dimensões analíticas, designadamente a funcional, a física, a simbólica e a relacional. Contribuem para estes défices de integração socioespacial, o reduzido parque de alojamento social e o seu direcionamento quase exclusivo para as famílias insolventes, a edificação dos empreendimentos num contexto de desarticulação urbana e os limitados impactos dos programas, práticas e ações sociais e socioterritoriais incidentes nos bairros, no que se refere a contributos efetivos para a sua integração socioespacial.
\end{abstract}

Palavras-chave: Integração socioespacial; habitação social; bairro; políticas de habitação social.

ABSTRACT - SOCIO-SPATIAL INTEGRATION OF SOCIAL HOUSING NEIGHBOURHOODS IN THE LISBON METROPOLITAN AREA: EVIDENCES OF MICRO SEGREGATION. This paper reflects on the urban condition of social housing neighbourhoods in the Lisbon Metropolitan Area (LMA) with regard to the socio-spatial integration and their relations with urban development, public policies and local action strategies in the neighbourhoods. This study uses a multi-scalar perspective, which starts with an extensive analysis of social housing neighbourhoods in the LMA and is continued by an intensive

Recebido: maio 2017. Aceite: novembro 2017.

1 Investigadora Associada do Centro de Estudos Geográficos do Instituto de Geografia e Ordenamento do Território da Universidade de Lisboa, R. Branca Edmée Marques, 1600-276, Lisboa, Portugal. E-mail: marinacarreiras@campus.ul.pt 
study of three neighbourhoods located in different municipalities in the Greater Lisbon area. The research involved, in addition to the literature review, a combination of quantitative, qualitative and cartographic methods. The findings highlight several constraints in the socio-spatial integration of social housing estates at multiple geographic levels and confirm the socio-spatial inequalities as a multilevel phenomenon that is expressed in various analytical dimensions, namely the functional, physical, symbolic and relational ones. Contributing to this socio-spatial integration deficit are the reduced number of social housing and the almost exclusive orientation towards insolvent households, the insertion of the neighbourhood in a context of urban disconnection and the limited impact of programs, practices and actions of social and socio-territorial nature implemented in neighbourhoods, regarding their effective contribution to socio-spatial integration.

Keywords: Socio-spatial integration; social housing; neighbourhood; social housing policies.

RÉSUMÉ - L'INTÉGRATION SOCIO-SPATIALE DES QUARTIERS DE LOGEMENTS SOCIAUX DANS L'AIRE URBAINE DE LISBONNE: LES ÉVIDENCES DE MICRO-SÉGRÉGATION. Cette étude traite des rapports que cette intégration maintient avec les caractères de l'urbanisation, des politiques publiques et des stratégies locales d'action. L'approche utilisée est multi-scalaire et a comme point de départ une analyse extensive des quartiers de logements sociaux, pour arriver à l'étude intensive de trois quartiers, situés dans différentes communes du Grand Lisbonne. On a eu recours, pour cela, après une analyse bibliographique exhaustive, à diverses méthodes quantitatives, qualitatives et cartographiques. On a ainsi mis en évidence, à divers niveaux géographiques, l'insuffisance de l'intégration socio-spatiale d'initiative publique dans ces quartiers. Ce qui confirme que les inégalités socio-spatiales se manifestent à divers niveaux analytiques : fonctionnels, physiques, symboliques et relationnels. Comme contribution à l'intégration socio-spatiale de ces quartiers, on signale les divers facteurs qui contribuent à leur déficit d'intégration: un nombre réduit de logements sociaux, presque tous réservés aux familles insolvables, l'implantation des cités dans un contexte de désarticulation urbaine et l'impact limité des programmes, des pratiques et des actions sociales et socio-territoriales, mis en œuvre dans ces quartiers.

Mots clés: Intégration socio-spatiale; logements sociaux; quartiers; politiques de logement social.

\section{INTRODUÇÃO}

As teorias e os debates associados às desigualdades socioespaciais em confronto com os padrões de desenvolvimento do espaço urbano e com as alterações nos modelos de organização económica e social são diversos (Harvey, 1990; Sassen, 1991; Smith, 1996; Short, 1996; Barata Salgueiro, 1999; 2000; Brenner \& Theodore, 2002; Malheiros, 2007) e evidenciam uma persistência, segmentação e complexidade das desigualdades socioespaciais.

Neste estudo, incidente sobre a temática das desigualdades socioespaciais, problematiza-se e analisa-se a questão da integração socioespacial dos bairros de habitação social. Essa foi uma escolha ponderada que resultou do interesse pela vulnerabilidade destes 
bairros e no papel potencial que a sua estrutura física e a sua localização relativa desempenham no agravamento dessa vulnerabilidade.

Os bairros de habitação social aparecem como espaços excluídos e estigmatizados, áreas de concentração de situações sociais desfavoráveis e de grupos étnicos minoritários, potencialmente geradoras de elevado risco social (Wacquant, 1996; Barata Salgueiro, 2000; Malheiros, Mendes, Barbosa, Silva, Schiltz, \& Vala, 2007). Isto é verificável no contexto português, no qual os "bairros sociais" são apontados como exemplos de espaços segregados do ponto de vista socioespacial, representando situações de imposição de segregação devido à concentração de populações mais desfavorecidas, sem ou com limitada capacidade de escolha do local de residência.

Os debates em torno das políticas de habitação são especialmente pertinentes no atual contexto de crise económica associada ao agravamento das necessidades sociais. Ainda que o atual parque habitacional social existente em Portugal seja parco comparativamente a outros países da União Europeia (Pittini, Ghekière, Dijol, \& Kiss, 2015) estes alojamentos, enquanto unidades espaciais de promoção pública, correspondem a uma materialização física e territorial direta das políticas de habitação. Permitem, por isso, realizar uma reflexão quanto à lógica que está por trás da integração e, também, quanto ao modo como os valores, normas e contextos (sociais, ambientais, históricos, culturais) se refletem nos resultados e nas práticas efetivas dessas políticas.

O trabalho apresentado tem por objetivo principal refletir sobre a persistência de desigualdades sociais e a sua expressão espacial através da análise dos bairros de habitação social. Em termos concretos procura-se: a) refletir sobre a integração socioespacial dos bairros de habitação social, através da identificação e sistematização de padrões de integração socioespacial; b) explorar relações entre a integração socioespacial dos bairros, o desenvolvimento urbano e as políticas públicas, principalmente as de habitação e ordenamento do território; c) e refletir sobre estratégias e ações territorializadas com eventual incidência nos bairros e seus impactos.

Tal ambição é alcançada através de estudo empírico desenvolvido em 2 níveis de análise geográfica, com recurso a pesquisa bibliográfica, tratamento e análise de dados estatísticos. São fontes secundárias as estatísticas do Instituto Nacional de Estatística (INE), em especial os Censos e o Inquérito à caracterização da habitação social (ICHS). A recolha direta de informação foi enquadrada no âmbito do projeto de investigação científica REHURB, sendo constituída por dados provenientes de: a) questionário sobre bairros de habitação social efetuado às 18 câmaras municipais da AML; b) inquéritos por questionário a 937 residentes nos três bairros que constituem estudos de caso; c) entrevistas a agentes chave e d) informação recolhida no âmbito de observação direta da vida nos bairros e das práticas sociais e espaciais da sua população.

$\mathrm{O}$ artigo está estruturado em 4 secções. Na primeira, fundamenta-se teórica e conceptualmente o recurso ao termo "integração socioespacial", ideia-chave que suporta a pesquisa. Na segunda secção descreve-se a incidência do princípio da integração socioespacial nas políticas de habitação social, e outras relacionadas, em Portugal. Na terceira secção, de natureza empírica, relatam-se os procedimentos metodológicos desenvolvidos 
e discutem-se os resultados da análise extensiva referente aos bairros de habitação social na AML assim como da investigação de âmbito intensivo incidente sobre três bairros de habitação social. Finalmente sintetizam-se criticamente os principais resultados da investigação.

\section{DIVERSIDADE URBANA E INTEGRAÇÃO SOCIOESPACIAL: NOTAS PARA DISCUSSÃO}

Os processos globais induzem alterações nas estruturas económicas, na composição social e étnica das populações, na cultura urbana, afetam as cidades, traduzindo-se em manifestações espaciais de divisão e aumento das desigualdades sociais, associadas ao desenvolvimento da organização social e espacial em rede (Viard, 1994; Malheiros, 2001), e à fragmentação e a ambiguidade do espaço urbano (Barata Salgueiro, 2000).

Por outro lado, os contextos e preexistências espaciais, estruturas sociais e políticas locais modelam e adaptam os efeitos da globalização (Maloutas, 2012). Ou seja, as dinâmicas e novos modos de organização urbanos não traduzem um desaparecimento completo das lógicas anteriores. Verificam-se situações de transição, complexificação das formas e dos padrões espaciais novos e pré-existentes, seja pela continuação de tendências antigas como pelo surgimento de novas forças (Beauregard \& Haila, 2000). Assim será adequado referir a ideia de diversificação (Préteceille, 2006) e não de homogeneidade de situações ou uma "nova ordem espacial" (Marcuse \& Kempen, 2000). Nesta sequência, os fenómenos de crescente polarização social, de fragmentação espacial e da emergência de novos padrões espaciais conduzem a desafios de reinterpretação dos conceitos teóricos que surgem como paradigmas das desigualdades socioespaciais tais como segregação, exclusão, fragmentação (adjetivados ou não com o termo socioespacial) e de outros conceitos como inserção, inclusão, integração, coesão social, direito à cidade e justiça espacial, aos quais é atribuído uma natureza reivindicativa. Harvey (2008), Marcuse (2009), Borja (2010), Soja (2010) e Lefebvre (2012), são apenas alguns dos autores que advogam uma cidade justa, acessível, participada.

No entanto, a generosidade destes fins traduz-se em dificuldades de operacionalização dos conceitos, nomeadamente na sua aplicabilidade política. Perante obstáculos diversos, admite-se que o desígnio do "direito à cidade" aplicado a uma situação concreta não irá remeter para uma situação ideal mas deverá abranger boas práticas com diferentes níveis de impacto na qualidade de vida dos utilizadores de determinado espaço (Sugranyes \& Mathivet, 2011, p. 129-192). Assume-se a igualdade territorial como utopia, concorrendo para tal fatores sociais, económicos e ambientais, notando-se que os lugares não são isotrópicos. Assim chega-se à conclusão que os espaços urbanos não têm de ser idênticos, seja na sua composição social, ou imagem urbana ou composição espacial. Advoga-se então a ideia de diversidade, diferenciação territorial e sobreposição, entendida como contributo para um maior grau de acessibilidade e interação, perspectiva que recupera elementos avançados por Jane Jacobs (1961) em meados do século XX. As 
subdivisões, em tempo ou espaço, são necessárias para uma referenciação e uma acomodação de vários usos e diferentes categorias de utilizadores (Kärrholm, 2007).

A diversidade espacial e social na cidade, seus impactos e consequências, remete para uma multiplicidade de perspectivas e para a reflexão teórica em torno da dialéctica socioespacial mas não se pretende neste texto questionar a relação que se considera indissociável entre dinâmicas sociais e espaciais (Comtois, 1986; Barata Salgueiro, 2002; Soja, 2010). Procura-se debater e apresentar caminhos para a análise de situações de exclusão e estigmatização urbana.

Os "bairros sociais", objeto de estudo aqui apresentado, são apontados como territórios excluídos e estigmatizados. Simultaneamente estas unidades espaciais de promoção pública permitem realizar uma reflexão quanto à integração, noção inerente às políticas habitacionais e urbanas portuguesas (Menezes \& Almeida, 2006).

A integração, neste estudo é entendida como integração socioespacial, conceito que procura representar de forma abrangente a ideia de construção de uma sociedade inscrita num espaço urbano inclusivo, conjugando as dimensões espacial e social.

O termo integração, com origem na disciplina da biologia, é utilizado no pensamento social a partir de finais do século XIX, período de desenvolvimento industrial, crescimento urbano e de situações de injustiça social, de marginalidade e de desigualdades sociais nas cidades. Émile Durkheim (2005 [1897]) surge como figura central para a compreensão das primeiras interpretações da integração enquanto sentimento de pertença de um individuo a um determinado grupo social (Ferreira, Peixoto, Carvalho, Raposo, Graça, \& Marques, 1995; Rhein, 2002).

Posteriormente na compreensão da integração, inicialmente de âmbito social e cívico, a dimensão espacial foi adquirindo maior expressão, até porque a consciência de que os défices sociais específicos têm uma expressão territorial e se manifestam nos lugares foi crescendo no âmbito dos estudos urbanos. Repare-se ainda que não são somente os indivíduos e grupos de indivíduos que se constituem como unidades de análise nos estudos de integração. A noção de integração é igualmente aplicada aos territórios conforme Barata Salgueiro (2000) concretiza quando apresenta processos de marginalização de território ou quando a União Europeia advoga uma ideia de coesão territorial (De Boe, Grasland, \& Healy, 1999).

A integração socioespacial surge enquanto conceito positivo e não proibitivo (Marcuse, 1997) como é o caso da segregação. Para além disso partilha um carácter reivindicativo como a noção de direito à cidade e apresenta-se como um conceito mais operativo, questão essencial para o presente estudo.

Para a operacionalização da ideia de integração considera-se como ponto de partida a exposição de Ruiz-Tagle (2013). Este autor entende integração socioespacial como a inclusão de um indivíduo na sociedade, limitando as desigualdades e situações de exclusão. Trata-se de uma noção compósita, que integra macro dimensões de análise espaciais e sociais, cuja natureza efetiva remete para o efeito do espaço nas relações sociais e, de forma mais específica, para o efeito mediador daquele. Neste âmbito a integração socioespacial remete para a eliminação de barreiras e/ou promoção de conexões, assim como 
para o reconhecimento da existência de canais de comunicação e de ligações (físicas, sociais, simbólicas, visuais, etc.) entre diversos grupos e territórios. Considera-se ainda que a operacionalização da noção de integração socioespacial deverá permitir uma leitura dinâmica e multinível do processo que envolva uma leitura multiescalar de um território (inserindo-o na sua envolvente próxima e, também, em estruturas mais amplas como o município, a região e o país) ou do confronto entre a situação de um grupo específico (a população em análise) face a outras populações.

Nestes moldes a integração socioespacial plena remete para um modelo idealizado de espaço (social) integrado, identificado e usado como plataforma de convivência social, de participação pública e de acesso a recursos e bens necessários a um pleno exercício da cidadania, posicionado de forma inequívoca e equilibrada numa rede espacial mais ampla.

\section{CONSIDERAÇÕES SOBRE O CONTEXTO PORTUGUÊS}

Em Portugal a integração socioespacial não assume primazia, mas é indiciada nas políticas públicas e é entendida como um princípio orientador nas áreas da habitação e no âmbito do ordenamento do território e urbanismo, nomeadamente através da dimensão social do planeamento urbanístico.

Não obstante os direitos consagrados na Constituição da República Portuguesa relativos à habitação e urbanismo (artigo $65^{\circ}$ ), o peso das políticas públicas de habitação em Portugal tem sido reduzido e a habitação social não assume no passado, nem atualmente, um papel de destaque na resolução dos problemas habitacionais (Serra, 2002; Nunes, 2011). A desregulação do sector habitacional e a subvalorização do caracter social do sector da habitação contribuíram significativamente para a persistência das carências habitacionais (Ferreira, 1987; Guerra, 1994; Serra, 2002; Menezes \& Almeida, 2006).

A fragilidade e insuficiência dos programas e estratégias de habitação, em especial no que se refere à intervenção direta do Estado, são reportadas por diversos autores. A título de exemplo, destacam-se os trabalhos de Bandeirinha (2007a), referentes à situação habitacional nos anos sessenta e de Pinto \& Guerra (2013) incidentes sobre as políticas de habitação social no período mais recente. Uma exposição mais extensiva dos programas de habitação social é realizada por Gros (1994) e por Serra (2002).

O setor da habitação social, nunca proveu um acesso generalizado à habitação, tendo sido no período anterior à revolução de 25 Abril de 1974 direcionado para o apoio a grupos sociais que se integravam no esquema corporativo e, posteriormente, restrito às famílias em situação de carência acentuada, nomeadamente aos núcleos familiares residentes em fogos de construção precária. Neste sentido, o período revolucionário foi excepcional e gerador de mudanças no setor da habitação de promoção pública (Portas, 1997). A experiência conhecida como SAAL (Serviço de Apoio Ambulatório Local) tornou-se paradigmática ao constituir-se como um exercício de democracia directa no qual os moradores eram parceiros de equipas técnicas pluridisciplinares (sobre esta prática consultar Bandeirinha, 2007b). 
No período pós-revolução a administração central reduz o seu papel na promoção e distribuição de alojamento. Há um desenvolvimento exponencial do mercado imobiliário privado, incentivado pela promoção da aquisição de casa própria através do sistema de crédito à casa própria. No entanto, esta nova lógica não constitui uma resposta para o acesso a uma habitação condigna por parte das famílias mais carenciadas e para a erradicação de bairros de barracas. A resolução das condições de habitação muito precárias das famílias implicará uma política de realojamento, sendo exemplo disto o PER (Programa Especial de Realojamento), programa destinado a extinguir os "conjuntos" de alojamentos precários nas áreas metropolitanas de Lisboa e do Porto, seja através de promoção municipal direta, seja através da promoção privada. O encerramento do ciclo construtivo associado ao PER precedeu o actual momento de redução de investimento na promoção pública, manutenção e gestão do parque de habitação social.

Considerando-se os vários programas de habitação social implementados reflecte-se sobre as orientações que ditaram a conceção dos bairros de habitação social em Portugal e explora-se o modo como essas intenções se concretizaram. Esta análise, conforme se verá na secção IV, incide nos conjuntos urbanos identificados como bairros de habitação social pelas autarquias.

A habitação social apresentou, regra geral, uma dimensão pouco lucrativa, não se tornando apelativa à intervenção dos privados. Por outro lado os custos controlados ${ }^{\mathrm{i}}$ condicionam a localização do empreendimento, a qualidade construtiva e as soluções construtivas. Estes aspetos são determinantes na geração de segregação socioespacial e de marginalização dos bairros de habitação social e confirmam a ideia da habitação enquanto pilar fraco do estado social (Torgerson, 1987) e da persistente ocorrência de desigualdades de base territorial (Pereira \& Ramalhete, 2017).

Adicionalmente as políticas de realojamento têm sido pautadas por incoerências, nomeadamente na resposta desproporcional entre provisão de alojamento (com maiores investimentos e impactos mais visíveis) e as estratégias de inclusão social (processos que implicam uma intervenção a longo prazo e remetem para impactos com menor visibilidade) das populações a realojar. $O$ alerta de necessidade de articulação entre a emergência da demolição de barracas e de construção de novos alojamentos com os processos de intervenção e acompanhamento social da população, necessariamente mais morosos, foi dado por Guerra (1994). Decorridas algumas décadas identificam-se erros cometidos, nomeadamente situações de deslocalização e dispersão de populações (Cachado, 2013; Ascensão, Colombo, Estevens, \& Ferreira, 2017).

\section{INTEGRAÇÃO SOCIOESPACIAL DE BAIRROS DE HABITAÇÃO SOCIAL}

No estudo de base empírica, o trabalho foi desenvolvido em duas fases, incidentes sobre duas escalas geográficas: metropolitana/municipal e local (bairro).

A análise extensiva dos bairros de habitação social à escala metropolitana e municipal, realizou-se tendo por base os dados recolhidos junto das autarquias. Foi ainda consi- 
derada literatura sobre as políticas de habitação social e o desenvolvimento urbano em Portugal. A segunda fase constituiu-se como uma análise intensiva incidente sobre 3 grandes bairros de habitação social, realizada através de estratégias diversas: inquéritos aos residentes, entrevistas, observação participante, análise documental e análise cartográfica.

\section{Bairros de habitação social na AML}

Para o universo de $237^{\text {ii }}$ bairros de habitação social identificados na AML, foram elaboradas análises que procuraram identificar padrões de integração socioespacial dos bairros no contexto metropolitano e municipal e assinalar eventuais relações entre as tipologias identificadas e os principais programas de habitação social.

Os bairros de habitação social foram georreferenciados ${ }^{\text {iii }}$ e analisados em duas escalas de análise. Uma primeira, municipal, incide sobre a localização do bairro à escala concelhia tendo-se observado três fatores: a) localização do bairro face aos limites administrativos do município em que este se insere; b) classificação do solo no qual o bairro se encontra implantado segundo o PDM (Plano Diretor Municipal) aplicável; c) densidade populacional da freguesia em que o bairro se localiza. A segunda, denominada de escala local, tendo por recurso imagens de satélite, foca a estrutura urbana em que os bairros se inserem, características dessa malha urbana e situações de (des)continuidade urbana do bairro face à envolvente.

Para cada bairro foi indicada a prevalência de uma de três condições no âmbito das duas escalas: a) tendência de integração, b) tendência de não integração ou c) dados não conclusivos. Do confronto entre as duas foram definidas quatro tipologias de localização dos bairros relativamente à integração socioespacial, expostas no quadro I.

Quadro I - Tipologia de bairros quanto à integração socioespacial.

Table I - Typology of neighbourhoods on socio-spatial integration.

\begin{tabular}{lccl}
\hline & Integração & $\begin{array}{c}\text { Integração } \\
\text { Escala local }\end{array}$ & \multicolumn{1}{c}{ Descrição } \\
\hline Tipo I & $\checkmark$ & $\checkmark$ & Bairro integrado \\
Tipo II & $\mathrm{X}$ & $\checkmark$ & Bairro micro segregado \\
Tipo III & $\checkmark$ & $\mathrm{X}$ & Bairro integrado em unidade urbana segregada \\
Tipo IV & $\mathrm{X}$ & $\mathrm{X}$ & Bairro segregado \\
Tipo V & Sem inf. & $\mathrm{X}$ & Tendência não integração à macro escala \\
Tipo VI & Sem inf. & $\checkmark$ & Tendência integração à macro escala \\
Inc. & Sem inf. & Sem inf. & Dados inconclusivos \\
\hline
\end{tabular}

$\checkmark$ - ocorre; X - não ocorre.

Quanto à distribuição geográfica dos bairros por tipologia (fig. 1) verifica-se uma relativa dispersão geográfica dos bairros em situação de micro segregação (tipo II) e uma concentração ou sobre representação dos bairros dos tipos III, IV e "não integração à 
macro escala", reconhecendo-se alguma coincidência com as áreas críticas urbanas identificadas no âmbito do Plano Regional de Ordenamento do Território da Área Metropolitana de Lisboa (CCRLVT, 2002, p. 26), o que reforça a identificação destes territórios como áreas desfavorecidas urbanística e socialmente. Nos municípios localizados a sul do rio Tejo, com menor número de bairros, não se assinalam concentrações de situações de não integração.

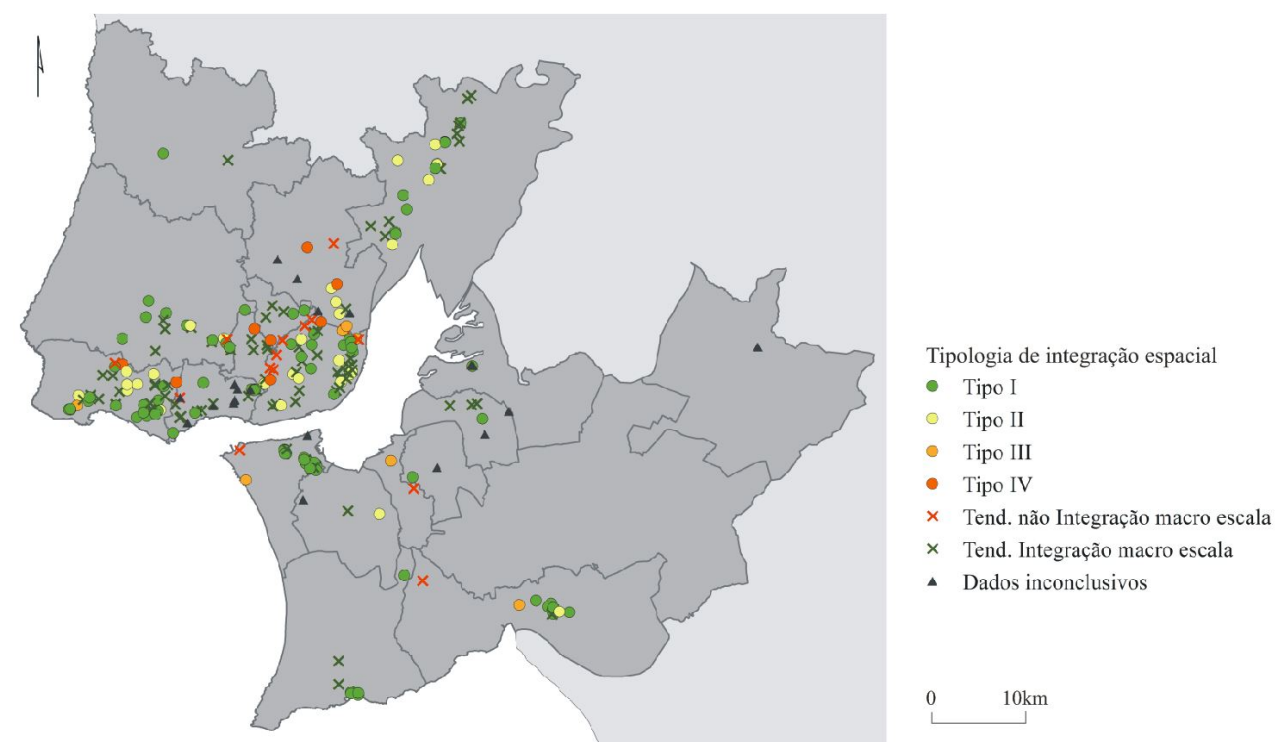

Fig. 1 - Bairros sociais na AML segundo tipologia de integração socioespacial.

Figura a cores disponível online.

Fig. 1 - Social housing neighbourhoods in LMA according to socio-spatial integration typology. Colour figure available online.

Retomando a reflexão sobre os impactos dos fenómenos da segregação e fragmentação urbana, pode-se extrapolar que os bairros de habitação social apresentam uma propensão para a integração urbana mais acentuada à macro escala, o que corrobora a significância do fenómeno de micro segregação no atual contexto dos bairros sociais da AML. Ou seja, as situações de integração socioespacial à escala metropolitana e municipal não implicam uma integração à escala do bairro e da sua envolvente.

Considerando-se a tipologia classificativa apresentada para os 237 bairros analisados (quadro II), ocorre uma tendência para a integração em 87 bairros, notando-se ainda que 86 bairros revelam tendência de integração à escala municipal, não existindo, para estes últimos, dados conclusivos à escala local. Nos resultados incidentes sobre a não integração socioespacial estão incluídos 61 bairros (29 tipo II - micro segregação, 8 tipo III integração em unidade urbana segregada, 8 tipo IV - segregação e 16 com tendência para não integração à macro escala). 
Analisaram-se ainda os níveis de integração socioespacial dos bairros considerando-se os programas de promoção de habitação social que lhes deram origem. Neste confronto (quadro II), verifica-se uma maior tendência para a integração socioespacial em todos os períodos temporais, sendo no entanto assinaláveis os fenómenos de micro segregação e de segregação nos bairros do período 3, e dos períodos 5 e 6.

Quadro II - Tipologia de integração socioespacial dos bairros vs. programas de habitação social.

Table II - Typology of neighbourhoods on socio-spatial integration vs Social housing programs.

\begin{tabular}{|c|c|c|c|c|c|c|c|c|}
\hline & & I & II & III & IV & V & VI & $\begin{array}{l}\text { Dados não } \\
\text { conclusivos }\end{array}$ \\
\hline \multirow{4}{*}{$\begin{array}{l}\text { Período } \\
\text { políticas } \\
\text { habitação }\end{array}$} & $\begin{array}{l}\text { Períodos } 1 / 2(\%) \\
N=29\end{array}$ & 51,7 & 3,4 & 6,9 & 0,0 & 10,3 & 27,6 & 0,0 \\
\hline & $\begin{array}{l}\text { Período } 3(\%) \\
\mathrm{N}=14\end{array}$ & 21,4 & 21,4 & 7,1 & 28,6 & 7,1 & 14,3 & 0,0 \\
\hline & $\begin{array}{l}\text { Período } 4(\%) \\
\mathrm{N}=45\end{array}$ & 33,3 & 8,9 & 2,2 & 4,4 & 4,4 & 46,7 & 0,0 \\
\hline & $\begin{array}{l}\text { Períodos 5/6 (\%) } \\
\mathrm{N}=76\end{array}$ & 28,9 & 15,8 & 1,3 & 1,3 & 9,2 & 40,8 & 2,6 \\
\hline \multicolumn{2}{|c|}{$\begin{array}{c}\text { Total de bairros } \\
\text { (valores absolutos) }\end{array}$} & 55 & 20 & 5 & 7 & 13 & 62 & 2 \\
\hline
\end{tabular}

Programas de habitação social por período:

1: Casas económicas, Casas famílias pobres, Casas de renda económica, Casas de renda limitada; 2: Planos integrados, FFH; 3: SAAL, CHE, PRID, Programa de empréstimos às câmaras, CAR; 4: PIMP; PRAUD; RECRIA; CDH; 5: PER, RECRIPH, PER Famílias; 6: PROHABITA

A primeira situação poderá ser, eventualmente, explicada pelos princípios orientadores de alguns dos programas habitacionais que advogavam um realojamento das populações no próprio local de residência (ex: Projeto SAAL, Serviço de Apoio Ambulatório Local) e pelo eventual défice em processos subsequentes de investimento e melhoria da qualidade urbana destes territórios. Os locais onde se construiu clandestinamente, localizavam-se normalmente em situação de periferia e/ou proximidade a territórios menos atractivos como áreas industriais, terrenos com declives acentuados, etc. A segunda situação remete para o grupo de bairros de promoção mais recente onde se incluem bairros abrangidos por iniciativas do PER ( $87 \%$ dos bairros deste grupo). A menor expressão de situações de integração neste conjunto de bairros é de certa forma prognosticada e identificada nalguns textos académicos desde inícios dos anos 90 do século XX (Cachado, 2013) quando a erradicação de bairros de barracas assumiu caracter de urgência e se prosseguiram estratégias de realojamento das populações assentes na construção intensiva de alojamento social.

Considera-se ainda que para alguns bairros, em especial para os mais antigos, a expansão e crescimento urbano das últimas décadas poderá ter contribuído para agregar e consolidar os tecidos urbanos, provocando um aumento da integração socioespacial, algo que será verificado, em mais profundidade, para os três casos de estudo. 


\section{Três casos de estudo}

A análise intensiva tem um caracter comparativo e incide sobre três bairros ${ }^{\text {iv }}$ de habitação social de grande dimensão localizados na metade norte da AML (fig. 2), construídos no mesmo período temporal (1990-2000), com número de habitantes superior a 900 e cuja construção teve por objetivo realojar famílias que residiam em condições precárias nos respetivos municípios (Páteo dos Cavaleiros em Oeiras, Casal dos Machados, em Lisboa, e Terraços da Ponte, em Loures).

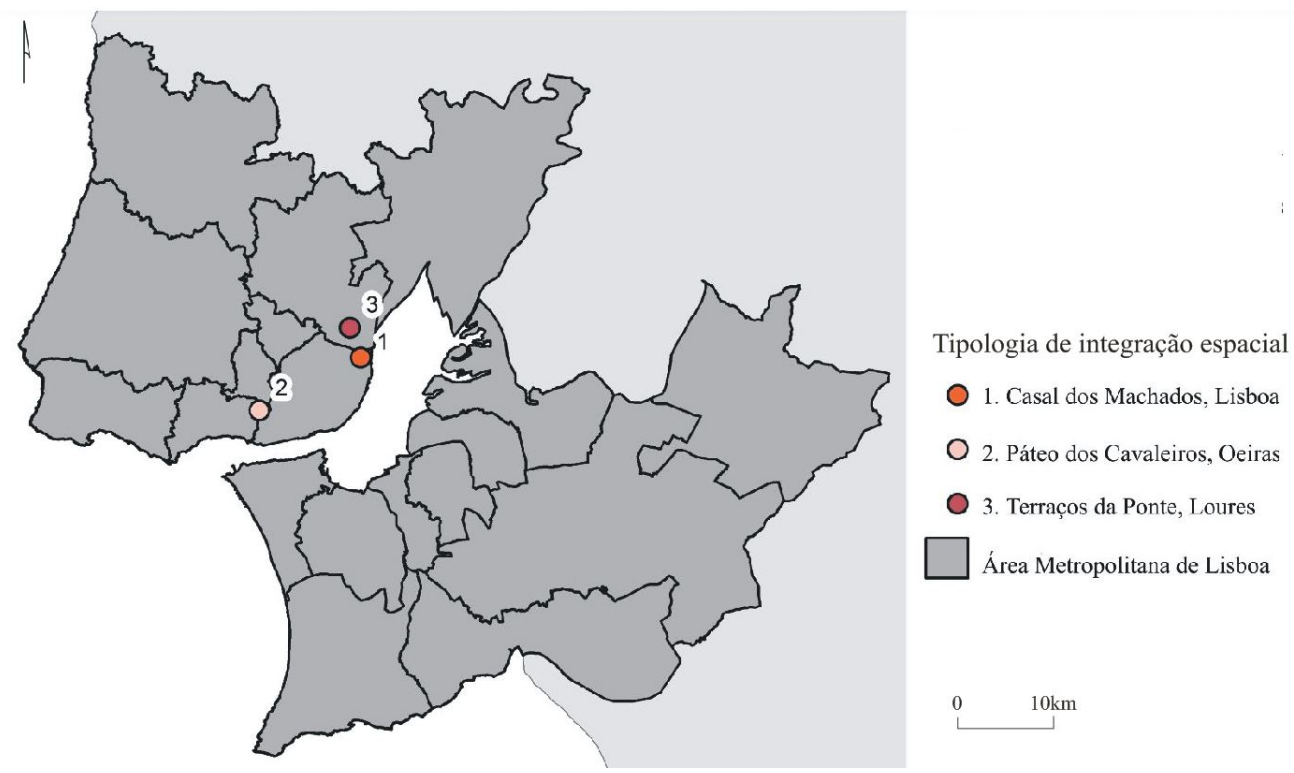

Fig. 2 - Localização dos bairros, casos de estudo, na AML. Figura a cores disponível online.

Fig. 2 - Location of neighbourhood, case studies, in LMA. Colour figure available online.

O estudo de cada uma das áreas foi realizado através de enquadramento territorial e histórico, caracterização física e funcional do bairro, análise das estratégias de gestão e de intervenção socio-urbanísticas no bairro e características sociodemográficas dos residentes. Considerou-se quer a perspetiva dos residentes, obtida através da aplicação de um questionário, quer a observação dos processos territoriais locais ao nível micro, por via de observação direta e continuada do espaço destes bairros e de entrevistas a dirigentes associativos e outros intervenientes nos processos. ${ }^{v}$ Os estudos tiveram por foco: (A) a integração socioespacial; (B) a evolução histórica da integração socioespacial; (C) a descrição e confronto dos instrumentos e estratégias com incidência nos bairros e eventuais impactos na integração socioespacial.

Para a análise da integração socioespacial (A) dos bairros consideram-se indicadores de pendor qualitativo, inseridos em quatro dimensões analíticas, classificados 
segundo uma escala ordinal de níveis de satisfação (quadro III). A avaliação da integração é concretizada através de posicionamento da situação dos três bairros por dimensão e subdimensão num referencial que varia entre cenários de desejabilidade e de marginalidade. Na sequência desta análise produziu-se uma síntese pluridimensional da integração socioespacial dos bairros (fig. 3), que reúne índices de integração por dimensão de análise, resultante da média dos valores das respetivas subdimensões de análise.

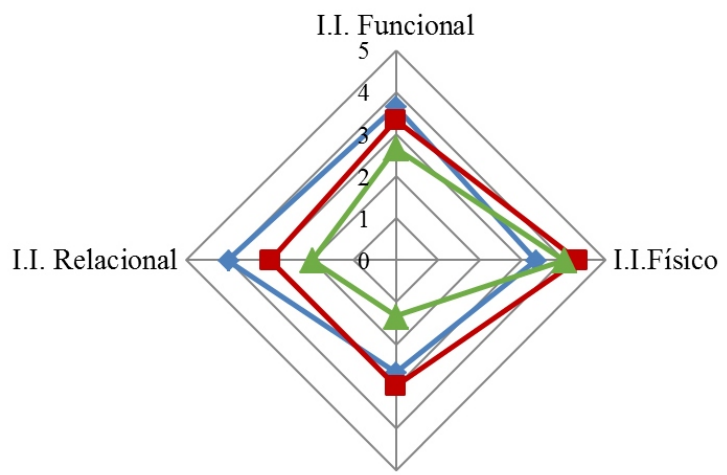

I.I. Simbólico

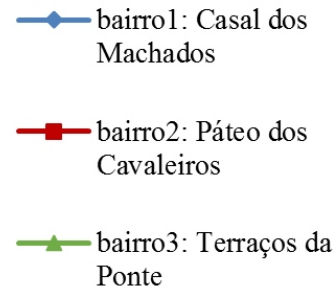
Ponte

Fig. 3 - Diagrama síntese comparativa da análise da integração socioespacial a nível local.

Figura a cores disponível online.

Fig. 3 - Diagram of comparative analysis of socio-spatial integration at the local level. Colour figure available online.

No conjunto do confronto dos níveis de integração socioespacial o Casal dos Machados e o Páteo dos Cavaleiros encontram-se numa situação mais favorável face aos Terraços da Ponte, principalmente no que se refere às dimensões simbólica e relacional. Neste aspecto são diversos os sinais que remetem para uma representação pouco positiva dos bairros, nomeadamente as perceções dos residentes quanto a sentimentos de segurança e a imagem visual dos bairros. Na dimensão relacional, o desfasamento dos índices é consequência de menor comunalidade entre os residentes nos bairros em análise e os residentes nas áreas envolventes e de menores afinidades institucionais com os territórios urbanos envolventes.

Ademais, tendo por recurso as observações realizadas ao nível metropolitano/municipal reflete-se sobre a integração socioespacial dos bairros numa perspectiva multiescalar. Mas será in situ que se detectam, com maior pormenor, deficiências e lacunas na integração socioespacial contrariando algumas visões iniciais de reconhecimento de integração socioespacial nestes bairros. Ou seja as análises mais detalhadas permitiram identificar insuficiências na integração socioespacial, que surgiram sucessivamente em maior número da escala metropolitana para a local. 
Quadro III - Situações extremas de integração socioespacial a nível local por dimensão de análise e escala de níveis de satisfação.

Table III - Extreme situations of socio-spatial integration at the local level by dimension and satisfaction scale.

\begin{tabular}{|c|c|c|}
\hline Dimensão e subdimensões & $\begin{array}{c}\text { Não integração } \\
\text { (100\% insatisfação) }\end{array}$ & $\begin{array}{c}\text { Integração } \\
(100 \% \text { satisfação) }\end{array}$ \\
\hline $\begin{array}{l}\text { FUNCIONAL } \\
\text { Rede viária } \\
\text { Rede pedonal } \\
\text { Transportes públicos }\end{array}$ & $\begin{array}{l}\text { Espaço de difícil acesso e percurso. } \\
\text { Inexistência ou reduzido número de } \\
\text { percursos pedonais, rede viária e } \\
\text { ciclovias: redes descontínuas, sem } \\
\text { ligação à envolvente e de má qualidade; } \\
\text { Impossibilidade de acesso a transportes } \\
\text { públicos. }\end{array}$ & $\begin{array}{l}\text { Espaço facilmente acedido e percorrido. } \\
\text { Diversidade e qualidade dos percursos } \\
\text { pedonais, rede viária e ciclovias que } \\
\text { permitem o acesso ao bairro e a sua } \\
\text { ligação à envolvente assim como o fácil } \\
\text { usufruto dos diversos espaços; } \\
\text { Facilidade e diversidade de acesso a } \\
\text { transportes públicos (presença no bairro; } \\
\text { até } 2 \text { minutos a pé) }\end{array}$ \\
\hline $\begin{array}{l}\text { FÍSICA } \\
\text { Equipamentos } \\
\text { Serviços e comércio } \\
\text { Espaços públicos e verdes }\end{array}$ & $\begin{array}{l}\text { Inexistência de espaços diversos que } \\
\text { proporcionem diversas vivências e } \\
\text { satisfação de necessidades básicas } \\
\text { (comércio, serviços, espaços verdes) ou } \\
\text { quando existentes em mau estado de } \\
\text { conservação, com má qualidade dos } \\
\text { serviços }\end{array}$ & $\begin{array}{l}\text { Abundância, diversidade e qualidade } \\
\text { de espaços que proporcionam vivências } \\
\text { e satisfação de necessidades básicas } \\
\text { (comércio, serviços, espaços verdes no } \\
\text { bairro e na área envolvente) }\end{array}$ \\
\hline $\begin{array}{l}\text { SIMBÓLICA } \\
\text { Imagem e design } \\
\text { Perceções de segurança } \\
\text { Imagem mediática }\end{array}$ & $\begin{array}{l}\text { Bairro e área envolvente com má } \\
\text { imagem (nos media e junto da } \\
\text { população). Sentimentos de } \\
\text { insegurança e desvalorização dos } \\
\text { espaços públicos e verdes } \\
\text { Desejos de abandono dos bairros por } \\
\text { parte dos seus moradores }\end{array}$ & $\begin{array}{l}\text { Bairro e área envolvente com boa } \\
\text { imagem. Espaços percecionados como } \\
\text { agradáveis e valorização dos mesmos } \\
\text { Espaço desejado pelos moradores e não } \\
\text { moradores }\end{array}$ \\
\hline $\begin{array}{l}\text { RELACIONAL } \\
\text { Comunalidade dos grupos } \\
\text { Interações }\end{array}$ & $\begin{array}{l}\text { Inexistência de interação ou ocorrência } \\
\text { de conflitos entre grupos residentes no } \\
\text { próprio bairro e, também, destes com } \\
\text { pessoas de áreas envolventes ou de } \\
\text { outras áreas da cidade }\end{array}$ & $\begin{array}{l}\text { Interação e elevada convivência entre } \\
\text { grupos de residentes no bairro e destes } \\
\text { com moradores de áreas envolventes e } \\
\text { mesmo outras áreas da cidade }\end{array}$ \\
\hline
\end{tabular}

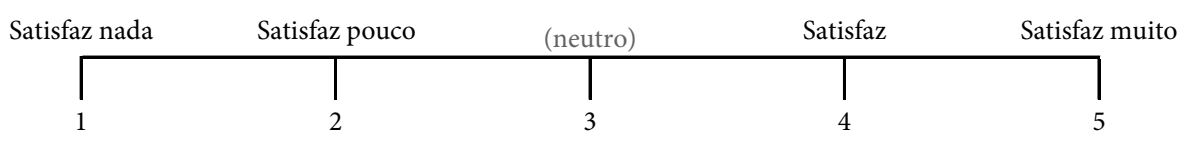

De uma forma geral, os três bairros apresentam uma situação vantajosa no panorama nacional ao localizarem-se na AML e, mais precisamente, em Lisboa ou na sua proximidade imediata. A infraestruturação existente no território da AML (redes viárias, redes de transportes públicos, redes de telecomunicações e energia, equipamentos das mais diversas áreas, serviços e comércio) proporciona facilidade de acesso a emprego, cuidados de saúde e outras necessidades básicas (educação, cultura, etc.).

A discussão dos níveis de integração socioespacial na escala municipal apresenta diversas complexidades pois os bairros inserem-se numa estrutura em rede na qual os 
territórios urbanos se estendem e se conectam sem relação direta com os limites administrativos. As tipologias, decorrentes da análise explanada na figura 1, atribuídas aos três bairros em estudo são para o Casal dos Machados: "Bairro integrado em unidade urbana segregada" e para o Páteo dos Cavaleiros e Terraços da Ponte: "Tendência integração à macro escala". Estas classificações não descrevem insuficiências que apenas são descobertas através de análise no local. Note-se, por exemplo que a estrutura de espaços coletivos (que compreendem a rede viária, os percursos pedonais e os espaços de acesso público no geral) dos bairros e áreas envolventes constituem situações de urbanização por enclave (processos de urbanização em que não são garantidas ligações aos territórios envolventes).

A evolução da integração do bairro (B) na estrutura urbana examina diferentes momentos temporais, através de recurso a informação cartográfica de cartas militares (Instituto Geográfico do Exército) e imagens satélite (Google Earth). Nos três casos de estudo verificam-se sinais de evolução e alteração dos níveis de integração socioespacial, nomeadamente através do crescimento urbano, da expansão das infraestruturas viárias e da rede de equipamentos, que nas últimas décadas veio beneficiar os bairros.

Os três bairros localizam-se em municípios distintos, todos com uma relevante presença de bairros de barracas na década de 80/90, tendo a sua extinção sido conseguida através de estratégias de realojamento associadas à construção de bairros de habitação social. Estas intervenções urbanas com mais restrições económicas do que os empreendimentos de promoção privada traduziram-se em localizações em terrenos municipais ou menos valorizados assim como em limitações quanto às áreas dos fogos e quanto ao valor dos materiais construtivos a usar.

O bairro localizado em Lisboa, apesar de ter sido construído num território bastante consolidado, veio a beneficiar, mais tarde, de novas infraestruturas implementadas aquando da promoção da Expo 98 e posteriormente da urbanização do Parque das Nações. O Páteo dos Cavaleiros, em Oeiras, terá sido aquele em que os níveis de integração na dimensão física e funcional menos mudou, contribuindo para tal o facto de ter sido uma das mais recentes operações urbanas a ser realizada na área. Dos três territórios em análise, o de Loures é aquele em que a ocupação urbana é mais recente sendo que a construção do bairro precedeu a construção de equipamentos de apoio à população assim como a construção do empreendimento privado contíguo (fig. 4).

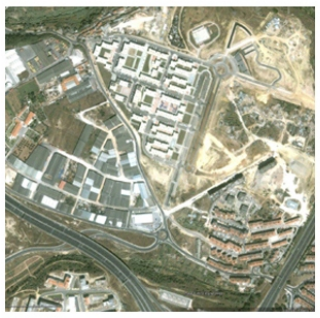

2001

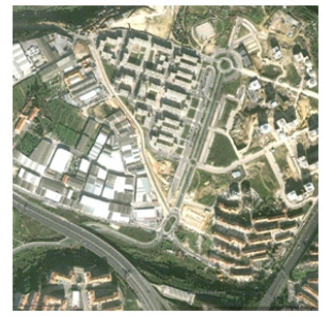

2005

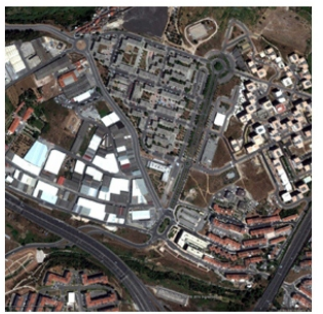

2012

Fig. 4 - Terraços da Ponte: Evolução da estrutura urbana. Figura a cores disponível online.

Fig. 4 - Terraços da Ponte: Evolution of the urban structure. Colour figure available online. 
A consideração dos instrumentos e estratégias com incidência nos bairros (C) teve em atenção: i) os programas de realojamento que lhes deram origem, ii) os Instrumentos de Gestão Territorial, em especial o PDM dos respetivos municípios e iii) outros planos/e estratégias de âmbito municipal e projetos ou ações de intervenção no bairro financiadas por programas nacionais ou comunitários promovidos isoladamente ou em parceria, já implementados, em vigor ou previstos para o futuro (quadro IV).

Quadro IV - Síntese de estratégias de intervenção assinaláveis nos bairros.

Table IV - Summary of intervention strategies in neighbourhoods.

\begin{tabular}{|c|c|c|c|c|}
\hline & & $\begin{array}{c}\text { Bairro 1 } \\
\text { Casal dos Machados }\end{array}$ & $\begin{array}{c}\text { Bairro } 2 \\
\text { Páteo dos Cavaleiros }\end{array}$ & $\begin{array}{c}\text { Bairro } 3 \\
\text { Terraços da Ponte }\end{array}$ \\
\hline \multicolumn{2}{|c|}{$\begin{array}{l}\text { Estratégias municipais } \\
\text { (ações extraordinárias }{ }^{\star} \text { ) }\end{array}$} & $\begin{array}{l}\text { Eventuais programas de } \\
\text { regeneração urbana no âmbito } \\
\text { do PDM; } \\
\text { Programa BIP/ZIP: } 0 \text { projetos; } \\
\text { PIGRBM }\end{array}$ & $\begin{array}{l}\text { Concretização das } \\
\text { intenções explanadas } \\
\text { no PP da Área Central } \\
\text { de Outurela-Portela }\end{array}$ & $\begin{array}{l}\text { Eventuais reconversões } \\
\text { das áreas envolventes } \\
\text { do bairro no âmbito } \\
\text { do PDM }\end{array}$ \\
\hline \multicolumn{2}{|c|}{$\begin{array}{l}\text { Programas com apoios } \\
\text { comunitários }\end{array}$} & ---- & $\begin{array}{l}\text { URBAN } \\
\text { PROQUAL (2001-2006), } \\
\text { inserido no QCA III }\end{array}$ & --- \\
\hline \multicolumn{2}{|c|}{$\begin{array}{l}\text { Programa Escolhas } \\
\text { (mais recente) }\end{array}$} & Entrelaços, és capaz! - E5G & ENTRECUL - E5G & $\begin{array}{l}\text { Projeto Esperança - } \\
\text { E5G }\end{array}$ \\
\hline \multicolumn{2}{|l|}{ Contexto escolar } & $\begin{array}{l}\text { AE Fernando Pessoa } \\
\text { TEIP desde 2009/10 }\end{array}$ & $\begin{array}{l}\text { AE Carnaxide-Portela } \\
\text { TEIP desde 2006/07 } \\
\text { Projeto Orquestra } \\
\text { Geração desde } 2010 / 11\end{array}$ & $\begin{array}{l}\text { AE Eduardo Gageiro } \\
\text { TEIP desde 2006/07 } \\
\text { Projeto Orquestra } \\
\text { Geração desde 2009/10 }\end{array}$ \\
\hline \multirow{2}{*}{$\begin{array}{l}\text { Grupos } \\
\text { comunitários } \\
\text { e outras } \\
\text { parcerias }\end{array}$} & 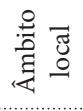 & $\begin{array}{l}\text { Rede L\&M no bairro do Casal } \\
\text { dos Machados e Quinta das } \\
\text { Laranjeiras }\end{array}$ & --- & --- \\
\hline & 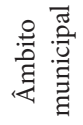 & Rede social de Lisboa & Rede social de Oeiras & $\begin{array}{l}\text { Rede social de Loures } \\
\text { Contrato Local de } \\
\text { Segurança }\end{array}$ \\
\hline \multicolumn{2}{|l|}{$\begin{array}{l}\text { Outros projetos } \\
\text { de intervenção }\end{array}$} & $\begin{array}{l}\text { Diversas atividades promovidas } \\
\text { pelas associações sedeadas no } \\
\text { bairro }\end{array}$ & $\begin{array}{l}\text { Diversas atividades } \\
\text { promovidas pelas } \\
\text { associações sedeadas na } \\
\text { Outurela-Portela }\end{array}$ & $\begin{array}{l}\text { Festival O bairro i o } \\
\text { mundo (2015) } \\
\text { Diversas atividades } \\
\text { promovidas pelas } \\
\text { associações sedeadas } \\
\text { no bairro }\end{array}$ \\
\hline
\end{tabular}

*Não se consideram neste quadro as ações gerais, inerentes à gestão do parque habitacional: apoio às famílias alojadas e pequenas intervenções de conservação e reparação nas habitações, espaços comuns dos edifícios e espaços públicos.

Os territórios onde os bairros se inserem são qualificados nos Instrumentos de Gestão Territorial de âmbito municipal como áreas urbanas consolidadas. No entanto, tal como já fora referido, verificam-se lacunas a nível da integração socioespacial, eventualmente de difícil quantificação ou com pouca expressão num "mundo fragmentado" mas que não passam despercebidos in loco, onde a separação entre os bairros analisados e outros territórios contíguos mais favorecidos é evidente. 
Os três bairros em análise apresentam diferentes características no que diz respeito à sua história, morfologia e inserção urbana, constituição sociodemográfica e diversidade étnica da população, mas todos remetem para situações de carência socioeconómica e desvantagens socioespaciais, sendo tal também comprovado pela diversidade e incidência de programas e ações que visam uma resolução para estes problemas.

\section{NOTAS FINAIS}

O trabalho apresentado evidencia o protagonismo de uma espacialidade das desigualdades. Não obstante, os princípios e desejos de integração socioespacial - presentes nos debates que evocam o direito à cidade, nas visões que apontam para a necessidade de estratégias progressivamente mais integradas, concertadas e sustentáveis - destaca-se a persistente incapacidade de colmatação das desigualdades socioespaciais, nomeadamente nas suas componentes espaciais.

O recurso ao conceito de integração socioespacial foi suportado pelo fato deste se constituir enquanto conceito positivo e não proibitivo, por englobar uma perspetiva de análise holística que contempla diversas dimensões de análise e por conciliar operatividade com uma dose de idealismo em linha com a ideia de direito à cidade. No entanto, denotou-se ao longo da investigação que o limitado e diversificado uso do termo em diversos trabalhos de pesquisa implicou experimentações na aplicação no estudo empírico e que a análise de múltiplos critérios, englobados na questão da integração socioespacial, é naturalmente dificultada pelas inter-relações entre os indicadores analíticos.

A literatura indica os contextos do realojamento das famílias carenciadas e de bairros de habitação social como exemplos da desconexão entre diversas dimensões, como a social, económica e a espacial. De facto, a ocorrência de integração socioespacial dos bairros de habitação social é desde logo comprometida quando se reconhecem como beneficiários da habitação de carácter social apenas as populações mais fragilizadas. Adicionalmente, a contemplação da ideia de integração socioespacial no processo de ordenamento do território e urbanismo, e mais especificamente no âmbito das políticas de habitação social, de uma forma geral, não garantiu uma efetiva situação de integração dos bairros e comunidades nas estruturas urbanas.

Mediante as análises realizadas, verifica-se que os bairros sociais na AML, no que se refere à sua dimensão espacial a uma escala metropolitana e municipal, encontram-se em situação menos desfavorável do que a indiciada na literatura. O desfavorecimento dos bairros de habitação social no sistema urbano português (isolamento, desconexão com áreas envolventes e fragmentação urbana), infelizmente, não representa uma exceção no contexto generalizado de desorganização territorial em Portugal. Assim, não são desprezáveis as situações de fragmentação urbana que se tornaram evidentes através da análise a uma escala local. $\mathrm{O}$ facto de a contiguidade não configurar continuidade urbana, conforme Barata Salgueiro (1999) explicita, nem indiciar interação entre populações vizinhas, é constatado no três bairros que constituem casos de estudo. Os resultados do estudo evidenciam a micro segregação destes territórios. 
A ocorrência de integração socioespacial surge dissociada dos processos de constituição dos bairros e de estratégias integradas. A integração socioespacial, nos três bairros analisados em profundidade, decorre em grande parte, de melhorias ocorridas desde o momento da sua conceção até ao momento atual, na sequência de dinâmicas distintas (construção de infraestruturas destinadas a outros territórios, inclusão numa estratégia de produção urbana mais abrangente e infraestruturação de emergência direcionada para as populações locais). De notar ainda que as lacunas identificadas nas dimensões de análise da integração socioespacial são mais evidentes na dimensão relacional e simbólica e menos nas dimensões espaciais (física e funcional). No contexto local, a imagem negativa do bairro, enquanto "bairro social", encontra-se patente nas perceções dos seus residentes e nas estratégias de ação social orientadas para aqueles territórios.

Finalmente reconhece-se que a exploração da situação de (des)integração socioespacial neste estudo, direcionada para os bairros de habitação social, poder-se-á estender a outro tipo de unidades urbanas, também segregadas e desarticuladas da envolvente, uma vez que esta situação não é exclusiva ao parque de habitação social. Entende-se ainda que a prossecução da ideia de integração socioespacial será particularmente relevante em contextos como o português, no qual os índices de segregação socioespacial são geralmente apontados como baixos, mas onde devido a diversos fatores, os empreendimentos urbanos, principalmente os mais recentes, não se encontram articulados de forma coerente numa rede urbana mais ampla.

Dada a dificuldade de valorização urbanística dos bairros sociais, e no atual contexto de implementação de intervenções urbanas pontuais e locais, o enfoque na integração socioespacial constitui um alerta para a necessidade de construção de estratégias de atuação, evidentemente integradas, que visem combater a fragmentação urbana e promover o direito à cidade na sua componente espacial.

\section{AGRADECIMENTOS}

O presente artigo apresenta contributos da Dissertação de Mestrado em Gestão de Território e Urbanismo intitulada "A integração socioespacial dos bairros de habitação social: um estudo da situação na Área Metropolitana de Lisboa” assim como do projeto de investigação REHURB - Realojamento e Regeneração Urbana (PTDC/CS-GEO/108610/2008).

\section{REFERÊNCIAS BIBLIOGRÁFICAS}

Ascensão, E., Colombo, A., Estevens, A., \& Ferreira, A. (2017). Splitering displacement: measuring the urban dispossession effects of the PER. Lisboa: Working Paper exPERts.

Barata Salgueiro, T. (2002). Espacialidade e temporalidades nas áreas urbanas [Spatiality and temporalities in urban areas]. In H. Cachi- nho, I. André, I. Medeiros, J. Reis, J. M. Malheiros, M. H. Esteves, R. J. Marques, S. Claudino, \& T. Barata Salgueiro (Eds.), Olhares sobre o território e a espacialidade [Looks over the territory and spatiality] (pp. 25-42). Lisboa: Centro de Estudos Geográficos da Universidade de Lisboa. 
Barata Salgueiro, T. (2000). Fragmentação e exclusão nas metrópoles [Fragmentation and exclusion in metropolises]. Sociedade e Território, 30, 16-26.

Barata Salgueiro, T. (1999). Ainda em torno da fragmentação do espaço urbano [Still around the fragmentation of the urban space]. Inforgeo, 14, 65-76.

Bandeirinha, J. A. (2007a). Anos sessenta. Verdades e consequências da crise da habitação em Portugal [Sixties.Truths and consequences of the housing crisis in Portugal]. Jornal Arquitectos, 226, 24-29.

Bandeirinha, J. A. (2007b). O Processo SAAL e a Arquitectura no 25 de Abril de 1974 [The SAAL process and architecture on April 25, 1974]. Coimbra: Imprensa da Universidade.

Beauregard, R., \& Haila, A. (2000). The Unavoidable Continuities of the City. In P. Marcuse, \& R. V. Kempen (Eds.), Globalizing cities: A new spatial order? (pp. 22-36). Oxford: Blackwell Publishing.

Borja, J. (2010). Democracy in Search of the Future City. In A. Sugranyes, \& C. Mathivet (Eds.), Cities for all: Proposal and experiences towards the right to the city (pp. 29-41). Santiago: Habitat International Coalition.

Brenner, N., \& Theodore, N. (Eds.). (2002). Spaces of Neoliberalism: Urban Restructuring in North America and Western Europe. Oxford: Blackwell.

Cachado, R. (2013). O Programa Especial de Realojamento. Ambiente histórico, político e social [Special Rehousing Program. Historical, political and social environment.]. Análise Social, XLVIII(206), 2182-2999.

Carreiras, M. (2015). A integração socioespacial dos bairros de habitação social: um estudo da situação na Área Metropolitana de Lisboa [The socio-spatial integration of social housing neighbourhoods: a study of the situation in the Lisbon Metropolitan Area] (Dissertação de Mestrado), Universidade de Lisboa. Retrived from http:// hdl.handle.net/10451/23146

Carreiras, M., Amílcar, A., Ferreira, B., \& Malheiros, J. (2011). Notas sobre a situação da habitação social em Portugal - Geografia, problemas e oportunidades? [Notes on the social housing situation in Portugal - Geography, problems and opportunities?]. Paper presented at the VIII Congresso da Geografia Portuguesa - Repensar a Geografia para novos desafios, Lisboa.

CCRLVT. (2002). PROT-AML Plano Regional de Ordenamento do Território da Área Metropolitana de Lisboa: Volume I (versão aprovada) [PROT-AML Regional Land Management Plan for the Lisbon Metropolitan Area: Volume I (approved version)]. Lisboa: Comissão de Coordenação da Região de Lisboa e Vale do Tejo.
Comtois, C. (1986). Spatial integration: review of theories and synthesis. Cahiers de géographie du Québec, 30(79), 41-51.

De Boe, P., Grasland, C., \& Healy, A. (1999). Spatial Integration. Strand 1.4 of the Study Programme on European Spatial Planning. Retrieved from http://www. mcrit.com/SPESP/SPESP_REPORT/1.4.\%20 Final\%20Report\%20(23-05-2000).pdf

Durkheim, E. (2005). [1897]. Suicide: A study in socio$\log y$. London and New York: Routledge.

Ferreira, A. (1987). Por uma nova política de habitação [For a new housing policy]. Porto: Edições Afrontamento.

Ferreira, J., Peixoto, J., Carvalho, A., Raposo, R., Graça, J., \& Marques, R. (Eds.). (1995). Sociologia [Sociology]. Lisboa: McGraw-Hill.

Guerra, I. (1994). As pessoas não são coisas que se ponham em gavetas [People are not things to put in drawers]. Sociedade e Território, 20, 11-16.

Gros, M. C. (1994). «Pequena» história do alojamentos social em Portugal [«Little» history of social housing in Portugal]. Sociedade e Território, 20, 80-90.

Harvey, D. (2008). The right to the city. New Left Review, $53,23-40$.

Harvey, D. (1990). The condition of postmodernity. Oxford: Blackwell.

Jacobs, J. (1961). The Death and Life of Great American Cities. New York: Random House.

Kärrholm, M. (2007). The Materiality of Territorial Production: A Conceptual Discussion of Territoriality, Materiality, and the Everyday Life of Public Space. Space and Culture, 10(4), 437-453.

Lefebvre, H. (2012). O direito à Cidade [The Right to the City]. Lisboa: Letra Livre.

Malheiros, J. (2007). Metropoles, dinâmicas económicas e reconfiguração espacial [Metropolises, economic dynamics and spatial reconfiguration]. In A. P. B. Horta (Ed.), Sociologia urbana [Urban sociology] (pp. 179-217). Lisboa: Universidade Aberta.

Malheiros, J. (2001). Arquipélagos migratórios: Transnacionalismo e Inovação [Migratory Archipelagos: Transnationalism and Innovation] (Tese de Doutoramento em Geografia), Lisboa: Universidade de Lisboa.

Malheiros, J., Mendes, M., Barbosa, C. E., Silva, S. B., Schiltz, A., \& Vala, F. (2007). Espaços e expressões de conflito e tensão entre autóctones, minorias migrantes e não migrantes na área metropolitana de Lisboa [Spaces and expressions of conflict and tension between autochtonous, migrant and non-migrant minorities in the Lisbon Metropolitan Area]. Lisboa: ACIME.

Maloutas, T. (2012). Introduction: Residential segregation in context. In T. Maloutas, \& K. Fujita (Eds.), 
Residential segregation in comparative perspective: Making sense of contextual diversity (pp. 1-36). Farnham: Ashgate.

Marcuse, P. (2009). From critical urban theory to the right to the city. City, 2-3, 185-197.

Marcuse, P., \& Kempen, R. v. (2000). Conclusion: A changed spatial order. In P. Marcuse, \& R. v. Kempen (Eds.), Globalizing cities: A new spatial order? (pp. 249- 275). Oxford: Blackwell Publishing.

Menezes, M., \& Almeida, A. (2006). Direito à cidade Reflexão em torno da incidência do termo mistura social nas politicas habitacionais e urbanas portuguesas [Right to the city - Reflection on the incidence of the term social mixture in Portuguese housing and urban policies]. Lisboa: LNEC.

Nunes, J. P. S. (2011). Florestas de cimento armado: Os grandes conjuntos residenciais e a constituição da Metrópole de Lisboa (1955-2005) [Forests made of reinforced concrete: Large residential developments and the constitution of the Metropolis of Lisbon (1955-2005)]. Lisboa: Fundação Calouste Gulbenkian.

Pereira, M., \& Ramalhete, F. (2017). Planeamento e conflitos territoriais: Uma leitura na óptica da (in) justiça espacial [Spatial planning and territorial conflicts: a reading from the spatial (in)justice perspective]. Finisterra - Revista Portuguesa de Geografia, LII (104), 7-24.

Pinto, T., \& Guerra, G. (2013). Some structural and emergent trends in Social Housing in Portugal. Rethinking housing policies in times of crisis. CIDADES, Comunidades e Territórios, 27, 1-21.

Pittini, A., Ghekière, L., Dijol, J., \& Kiss, I. (2015). The state of housing in the EU 2015. Retrived from https:// www.bwo.admin.ch/dam/bwo/de/dokumente/01 Wohnungsmarkt/16 Zahlen und Fakten/the state of housingintheeu2015.pdf.download.pdf/ the state of housingintheeu2015.pdf

Portas, N. (1997). A arquitectura da habitação no século XX Português [The architecture of housing in the Portuguese 20th century]. In A. Becker, A. Tostões, \& W. Wang (Eds.), Arquitectura do século XX: Portugal [20th Century Architecture: Portugal] (pp. 117-122). Frankfurt, Lisboa: Deutches Achitektur-Museum.

Préteceille, E. (2006). La ségrégation sociale a-t-elle augmenté? La métropole parisienne entre polarisation et mixité [Has social segregation increased? The Paris metropolis between polarization and diversity]. Sociétés Contemporaines, 62, 62-93.

Rhein, C. (2002). Intégration sociale, intégration spatiale [Social integration, spatial integration]. Espace géographique, 3(31), 193-207.

Ruiz-Tagle, J. (2013). A Theory of Socio-spatial integration: Problems, Policies and Concepts from a US Perspective. International Journal of Urban and Regional Research, 37(2), 388-408.

Sassen, S. (1991). The global city: New York, London, Tokyo. Princeton: Princeton University Press.

Serra, N. (2002). Estado, Território e Estratégias de Habitação [State, Territory and Housing Strategies]. Coimbra: Quarteto.

Short, J. R. (1996). The urban order: An introduction to cities, culture, and power. Oxford: Balckwell plublishers.

Smith, N. (1996). The New Urban Frontier: Gentrification and the Revanchist City. New York: Routledge.

Soja, E. (2010). Seeking Spatial Justice. Minneapolis: University of Minnesota.

Sugranyes, A., \& Mathivet, C. (Eds.). (2011). Cities for all: proposals and experiences towards the right to the city. Chile: Habitat International Coalition.

Torgerson, U. (1987). Housing: The wobbly pillar under the welfare state. In B. Turner, J. Kemeny, \& L. Lundqvist (Eds.), Between State and Market: Housing in the Post-industrial Era (pp. 116-127). Stockholm: Almqvist and Wiksell.

Viard, J. (1994). La Société d'archipel ou Les Territoires du village global [The archipelago society or the territories of the global village]. La Tour d'Aigues: L'Aube.

Wacquant, L. (1996). The Rise of Advanced Marginality: Notes on its Nature and Implications. Acta Sociologica, 39, 121-139.

i A concessão de apoios estatais à Habitação a Custos Controlados obedece a limites de área bruta, custos de construção e preço de venda.

ii Este número de bairros é decorrente de pedido de informação junto de todos os municípios da AML. Note-se que tendencialmente os municípios indicam os bairros de habitação social dos quais são proprietários, excluindo habitações que foram alienadas e fogos que são propriedade de organismos da administração central.

iii Consideraram-se os limites administrativos vigentes em 2011 e os ortofotomapas disponibilizados através do World Imagery Map Service.

iv A escolha dos bairros é coincidente com os casos de estudo do projecto REHURB e justificada pelos critérios de seleção do projeto incidentes na diversidade de situações que representam e nalgumas características chave que têm em comum: data de construção; grande dimensão à escala nacional, considerando-se o números de residentes; objetivos da promoção da edificação. A seleção dos bairros foi realizada na sequência de uma análise de correspondência múltipla seguida de uma análise de clusters e de visita in situ.

v Para um conhecimento mais aprofundado deste estudo consultar Carreiras (2015). 